\title{
Fondements Géographiques De La Valorisation Agricole Des Bas-Fonds Au Sud Du Bassin Versant De l'Oti (Bénin)
}

\author{
Kafilatou T. Souberou \\ Laboratoire Pierre PAGNEY Climat, Eau, \\ Ecosystèmes et Développement (LACEEDE), \\ Département de Géographie et Aménagement de Territoire (DGAT) \\ Imorou Ouorou Barre \\ Laboratoire Pierre PAGNEY Climat, Eau, \\ Ecosystèmes et Développement (LACEEDE), \\ Département de Géographie et Aménagement de Territoire (DGAT) \\ Université de Parakou (UP), Bénin

\section{Ibourä̈ma Yabi \\ Euloge Ogouwale} \\ Laboratoire Pierre PAGNEY Climat, Eau, \\ Ecosystèmes et Développement (LACEEDE), \\ Département de Géographie et Aménagement de Territoire (DGAT) \\ Université d'Abomey-Calavi (UAC)
}

Doi: 10.19044/esj.2018.v14n21p136 URL:http://dx.doi.org/10.19044/esj.2018.v14n21p136

\begin{abstract}
In the south of Oti catchment in Benin, the agricultural development of the lowlands for rice and vegetable cultivation, guarantees a large part of food resources. It also serves as an important source of financial income to agricultural households. This paper focuses on understanding the geographical foundations of this kind of agricultural production in the study area. Surveys of 207 farm households and the statistical processing of climatic, demographic, agricultural and socio-economic data have made it possible to highlight the natural and human factors of farm occupation and the use of these lowland agro-ecosystems. The results obtained revealed that the population of the south of Oti catchment has almost doubly increased between 1992 and 2013. It will increase four times in 2050 by creating the need to have excess food. In addition to this situation, climatic parameters fluctuation, plateau land qualitative tiredness, and major constraints of traditional agriculture were added. Henceforth, these are factors, in addition to the richness of the lowlands and water availability for a long period, which
\end{abstract}


have helped in motivating the population of farmers. About 2000 ha of lowlands were reclaimed by irrigation schemes between 1982 and 2015 in this area. This was possible with the help of technical structures and agricultural development organizations.

Keywords: Oti catchment, lowlands, utilization, hydroagricultural management, geographical foundations

\section{Résumé}

Dans le sud du bassin versant de l'Oti au Bénin, la mise en valeur agricole des bas-fonds pour la riziculture et le maraichage, assure une large part des ressources alimentaires et constitue une source d'importants revenus monétaires pour les ménages agricoles. La présente recherche vise à comprendre les fondements géographiques de cette forme de production agricole pratiquée dans la zone d'étude. Des enquêtes réalisées auprès de 207 ménages agricoles et le traitement statistique des données climatiques, démographiques, des productions agricoles et socio-économiques ont permis de mettre en évidence les facteurs naturels et humains de l'occupation et de l'utilisation de ces agroécosystèmes de bas-fonds. Les principaux résultats obtenus ont révélés que la population a presque doublé entre 1992 et 2013 et va quadrupler en 2050, constituant ainsi un surplus de besoins alimentaires à chercher. A cela s'ajoutent la fluctuation des paramètres climatiques et l'épuisement qualitatif des terres de plateaux, contraintes majeures pour l'agriculture traditionnel. Il ressort alors que ce sont ces facteurs, en plus, de la richesse pédologique des bas-fonds et la disponibilité de l'eau sur une longue durée qui conduisent la population paysanne à leur mise en valeur. Environ 2000 ha de bas-fonds ont été valorisés par des aménagements hydroagricoles entre 1982 et 2015 dans cette zone, avec le concours des structures techniques et organismes de développement agricole.

Mots-clés : Bassin versant de l'Oti, bas-fonds, exploitation, aménagement hydroagricole, fondements géographiques

\section{Introduction}

Ces deux dernières décennies, les communautés agricoles et les organismes de développement se sont évertués à développer face à la dégradation des sols (épuisement qualitatif et quantitatif des sols fertiles) et suite à plusieurs des modifications perçues dans les paramètres de la saison agricole des techniques ou pratiques. Elles se résument à la diversification des cultures, la mise en valeur des zones humides, l'introduction des plantes et arbres fertilisants des sols, la gestion de l'eau sur les exploitations (Abdou, 2016). Environ $46 \%$ de la population agricole au Bénin étendent leur 
exploitation agricole surtout vers les versants abrupts des cours d'eau et les bas-fonds en raison de l'absence, de l'insuffisance et de l'excès ou de la mauvaise répartition spatio-temporelle des pluies. Ce mouvement de la population est favorisé par les potentialités offertes par le milieu d'accueil, considérées comme des sols à hauts rendements agricoles. La mise en valeur des bas-fonds permet aux producteurs de tirer profit des potentiels pédologiques et hydrologiques disponibles et des opportunités socioéconomiques indéniables (Oloukoi et al., 2013)

Les bas-fonds, espaces multi-usages, considérés comme des milieux fertiles, moyennant des aménagements hydroagricoles portent des cultures permanentes. Ils constituent alors des pivots d'intensification agricoles (Lavigne Delville, 1996). Au vue ces intérêts potentiels, les producteurs s'intéressent de plus en plus à leur valorisation en adoptant des stratégies d'exploitation afin d'accroître la production agricole. Ceci se traduit par une expansion rapide des espaces agricoles et une régression des formations végétales de l'ordre de 0,38\% sous la période 1988-2015 dans le bassin versant de l'Oti qui est le secteur géographique de l'étude (Souberou et al., 2017). Une analyse des facteurs qui concourent à cette dynamique d'exploitation des bas-fonds s'impose pour une gestion durable de ces agroécosystèmes qui sont devenus outre le système de forte production agricole, un creuset de rivalité entre autochtones et migrants

La zone d'étude (Figure 1) est une partie du bassin hydrographique de 1'Oti qui s'étend sur le territoire de la République du Bénin (WHYCOS, 2006). Situé au nord-ouest du Bénin, elle est à cheval sur les Départements de 1'Atacora et de la Donga et occupe 47,20 \% de la superficie totale du bassin versant de l'Oti au Bénin soit environ 803411 ha. Comprise entre 09¹9'6', et $10^{\circ} 54^{\prime} 8^{\prime}$ ' de latitude nord d'une part et $0^{\circ} 45^{\prime} 34^{\prime}$ ' et $1^{\circ} 41^{\prime} 48^{\prime}$ ' de longitude est d'autre part, le sud du bassin versant de l'Oti est un milieu géographique original par son relief, sa forte densité de la population, sa richesse édaphique (sols minéraux bruts, sols ferrugineux tropicaux et sols ferralitiques), sa forte hétérogénéité sociale toujours en quête des terres fertiles pour l'agriculture (principale activité) et ses systèmes de cultures.

La géomorphologie révèle du fait de l'orographie, la présence d'un nombre important de zones humides. Ainsi, trois grandes unités morphologiques (chaîne de l'Atacora, de la pénéplaine et de la plaine de Gourma) échelonnant entre 118 et 667 m caractérisent cette partie (Agbossou \& Okoundé, 2001 ; Idiéti, 2012).

Sous l'influence du climat guinéo-soudanien et soudanien, cette zone est bien arrosée et le réseau hydrographique est dense. Deux grandes saisons rythment le développement des activités agricoles, il s'agit de la saison pluvieuse (mai à octobre) et la saison sèche (novembre à avril). La moyenne annuelle des précipitations se situe entre 967 et $1255 \mathrm{~mm}$ et peut atteindre 
parfois $1350 \mathrm{~mm}$ avec de fortes disparités temporelles et spatiales ces quatre dernières décennies (Ouorou Barrè, 2014). Pour ce qui est de la tendance thermométrique, il est caractérisé par trois périodes de chaleur. La première de forte chaleur (février-avril) entrainant l'assèchement des sols en hautes altitudes et amène la population à se précipiter vers les zones basses pour les activités de décrue. La deuxième de moyenne chaleur (mai-octobre) correspond à la saison pluvieuse et la troisième relativement faible (novembrejanvier) avec une fraîcheur relative. La température moyenne enregistrée sur la période de 1971 à 2014 est de $27,35^{\circ} \mathrm{C}$.

Au total, le sud du bassin versant de l'Oti (Figure 1), de par sa position géographique dispose quelques atouts (pédologique, hydrologique, etc.) qui suscitent la mise en valeur des bas-fonds.

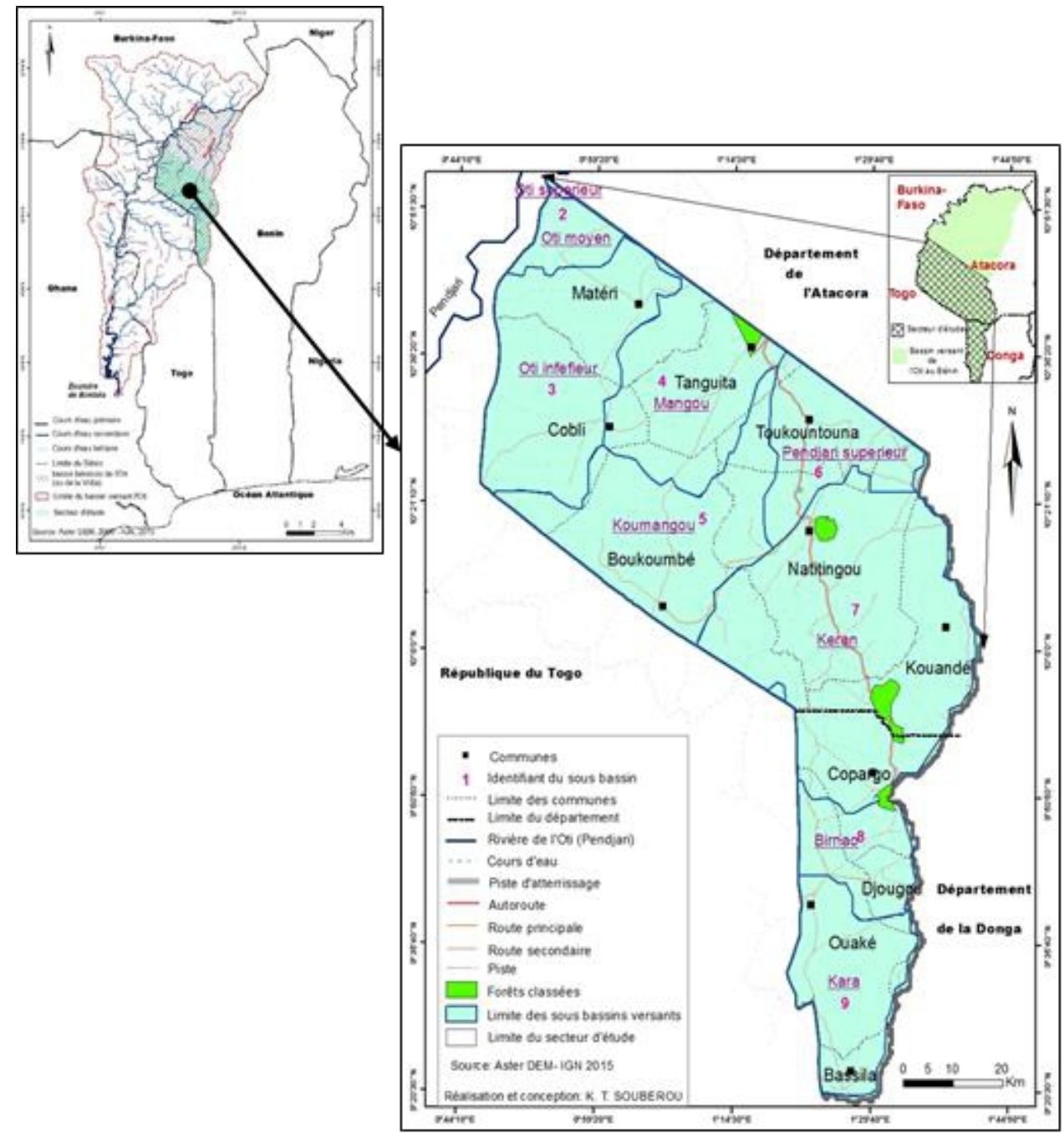

Figure 1. Situation géographique et administrative de la zone d'étude 


\section{Données et méthodes}

Les données utilisées sont multiples. Elles se rapportent essentiellement aux statistiques démographiques notamment les résultats des recensements de la population de 1979 à 2013, climatologiques à savoir les hauteurs de pluie, la température et l'Evapo Transpiration Potentielle pour la période 1971 à 2014 et agricoles que sont les (emblavures et productions des campagnes agricoles de 1995 à 2014). De même, les informations socioéconomiques qui se résument aux informations quantitatives et qualitatives ayant servir à appréhender les stratégies de mise en valeur des bas-fonds ont été collectées. Ces données sont obtenues respectivement à l'INSAE de Cotonou, à l'Agence pour la Sécurité de la Navigation Aérienne en Afrique et à Madagascar de Cotonou et sur le terrain à travers des entretiens directs, des observations directe comme technique de collecte de ces données.

A cet effet, un échantillon de 207 ménages agricoles repartis dans les 46 arrondissements des 11 communes (Bassila, Boukoumbé, Corpago, Cobly, Djougou, Kouandé, Matéri, Natitingou, Ouaké, Tanguiéta, Toukoumtouna) du sud du bassin versant de l'Oti à raison de 4 chefs de ménage par Arrondissement a été défini. La taille minimale de l'échantillon est calculée par la formule de Schwarzt (1995) : $\mathbf{n}=\left(\mathbf{t}^{2} \mathbf{x}\right.$ PQ) $/ \mathbf{e}^{2}$, avec $\mathbf{n}$ la taille de l'échantillon pour une population mère (nombre de ménages agricoles), $\mathbf{t}$ le coefficient de marge ( $\mathrm{T}$ de Student) déduit du Taux de confiance « $\mathrm{s}$ » ou le niveau de confiance que l'on souhaite garantir sur la mesure, $\mathbf{P}$ la proportion des ménages agricoles concernés par l'exploitation agricole des bas-fonds par rapport au nombre de ménages dans le milieu d'étude, $\mathbf{Q}=(\mathbf{1}-\mathbf{P})$ la probabilité d'échec ou probabilité de réalisation négative, e la marge d'erreur que l'on se donne pour la grandeur que l'on veut estimer (degré de précision avec une proportion réelle à $5 \%$ près donc e égal à 0,05 ). Le seuil de confiance est fixé ou réduit à un risque de $5 \%$ qui est une norme partagée (Durand, 2002 ce qui correspond à un degré de confiance de $95 \%$ pour le choix de l'échantillon aléatoire, donc $\mathbf{t}$ est égal à 1,96. Pour être choisi, le chef de ménage doit en outre être habitant de la localité, avoir au moins 30 ans, avoir exploité l'un des bas-fonds de la localité au moins ces 5 dernières années, avoir vécu dans l'Arrondissement et exercer l'activité agricole pendant au moins 30 ans (exploitant ayant une perception des changements climatiques, de l'évolution de la production agricole et des techniques de mise en valeur des bas-fonds).

Le choix de-92 bas-fonds visités a été fondé sur l'importance des activités agricoles et leur accessibilité.

Le traitement des données démographiques et climatologiques a nécessité l'utilisation des protocoles statistiques. L'estimation de la population à une date $\mathrm{t}+1$ a été réalisée à l'aide de la formule : $\mathbf{P}_{\mathbf{t}+\mathbf{1}}=\mathbf{P}_{\mathbf{t}}(\mathbf{1}+\mathbf{r})^{\mathbf{n}}$; avec $\mathbf{P}_{\mathbf{t}}$ $+\mathbf{1}$ la population projetée à la date $t+1, \mathbf{P}_{\mathbf{t}}$ la population de référence à la date $\mathrm{t}, \mathbf{r}$ le taux d'accroissement de la population entre $t$ et $\mathrm{t}+1, \mathbf{n}$ la différence entre 
l'année projetée et l'année de référence. Le calcul de la dispersion des valeurs autour de la moyenne a été effectué par la formule de la moyenne arithmétique (M) : $M=\frac{1}{n} \sum_{i=1}^{n} x i$, avec $\mathbf{n}$ l'effectif total des modalités et xi modalités du caractère étudié. Le calcul de l'indice pluviométrique $\mathbf{a}=(\mathbf{x i}-\mathbf{x m o y}) / \boldsymbol{\delta}$ avec xi la variable étudiée pour une année, xmoy la hauteur moyenne des pluies et $\boldsymbol{\delta}$ l'écart type de la série afin de caractériser les années excédentaires et déficitaires. L'analyse des bilans climatiques a permis de traduire le rythme des excédents et des déficits en eau au sud du bassin versant de l'Oti.

En ce qui concerne les informations agricole et socio-anthropologique, elles ont été traitées par l'utilisation des paramètres de la statistique descriptive (moyenne, fréquence et illustration graphique). Les taux des réponses ont permis de faire une analyse des facteurs endogènes de cette mutation agricole notamment la valorisation des bas-fonds.

\section{Résultats et discussion}

\section{Aperçu sur la valorisation des bas-fonds du bassin versant de l'Oti}

Les bas-fonds au sud du bassin versant de l'Oti sont liés aux axes de drainage fonctionnant plus en saison pluvieuse qu'en période sèche. Pour ce faire, des types d'aménagements hydroagricoles ont été mis en place pour mieux les exploiter. Ceux-ci concernent, les systèmes de pompage avec bassins ou puits pour arrosage manuel ou automatique des cultures (planche 1) ; les digues de protection perpendiculaires au sens d'écoulement de l'eau; les diguettes isohypses (principales et secondaires) en terre compactée et enherbée ou en pierres sèches (Cordons pierreux) de rétention, équipés d'ouvrages de vidanges ou d'un chenal central (planche 2) et; l'implantation de bandes enherbées de vétivers pour la réduction du ruissellement et facilitation de l'infiltration de l'eau. A cela s'ajoute la réalisation des casiers pour une bonne maitrise de l'eau.
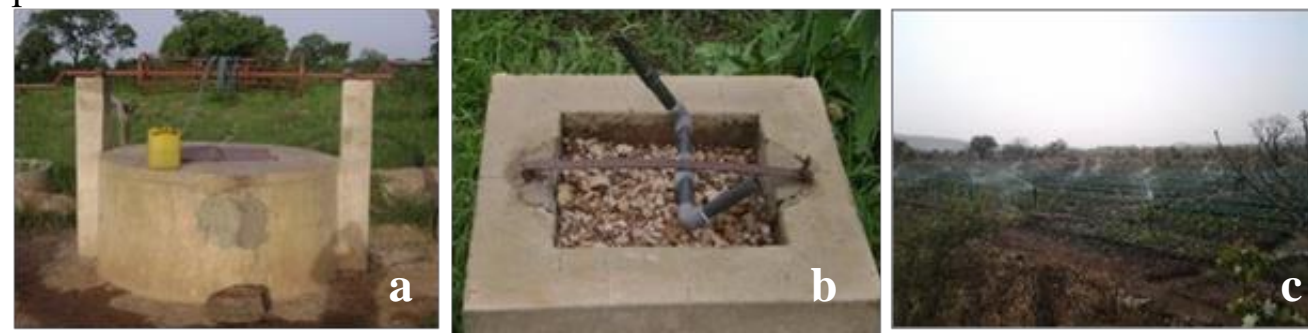

Planche 1. Puits maraicher munis d'un treuil à Firihoun et puits tubé à Lafirihoun (b) plus réseau d'irrigation (c) dans la commune de Matéri

La photo (a) de la planche 1 montre un puits à grand diamètre munis d'un système de treuil réalisé pour la production de contre saison. La photo (b) de la même planche montre un système de puits tubé qui permet de mobiliser l'eau souterraine à faible profondeur avec une motopompe pour 
irriguer l'exploitation agricole comme le présente la photo (c). Elle montre un système de tourniquets (réseau de canalisation alimentée sous pression avec des sprinklers) installé sur le site maraîcher de Lafirihoun. Ce système gravitaire permet d'améliorer le mode d'exploitation existant et de diminuer les corvées d'arrosage.

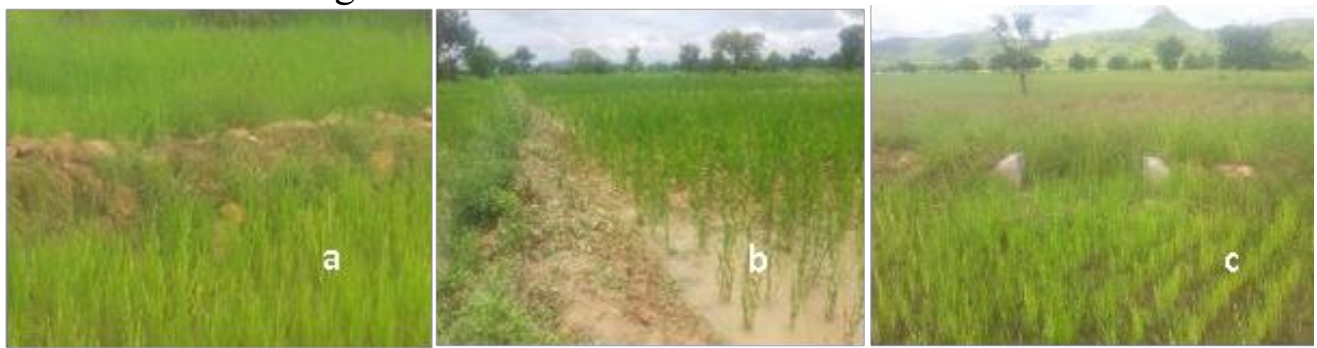

Planche 2 : Diguette d'amortissement en pierre (a), en terre (b) et ouvrage de vidange sans vanne (c) dans le bas-fond de Koubirgou de la commune de Boukoumbé

Les Photos ( $\mathrm{a}, \mathrm{b}$ et $\mathrm{c})$ de la planche 2 montre des aménagements réalisés qui sont constitué des diguettes d'amortissement en pierres, des diguettes principales et secondaires en terre et les ouvrages de vidange sans vanne qui jouent un rôle efficace dans la gestion de l'eau sur l'exploitation de production du riz.

Les bas-fonds sont exploités dans leur ensemble pour la riziculture (saison pluvieuse), le maïsiculture (saison pluvieuse), le maraîchage (saison pluvieuse et sèche) et autres cultures vivrières. La riziculture et le maïsiculture sont des activités tributaires de la quantité et de la répartition des pluies au niveau du bassin versant de l'Oti. Elles sont pratiquées en monoculture sur des sols à bonne capacité de rétention en eau (planche 3).

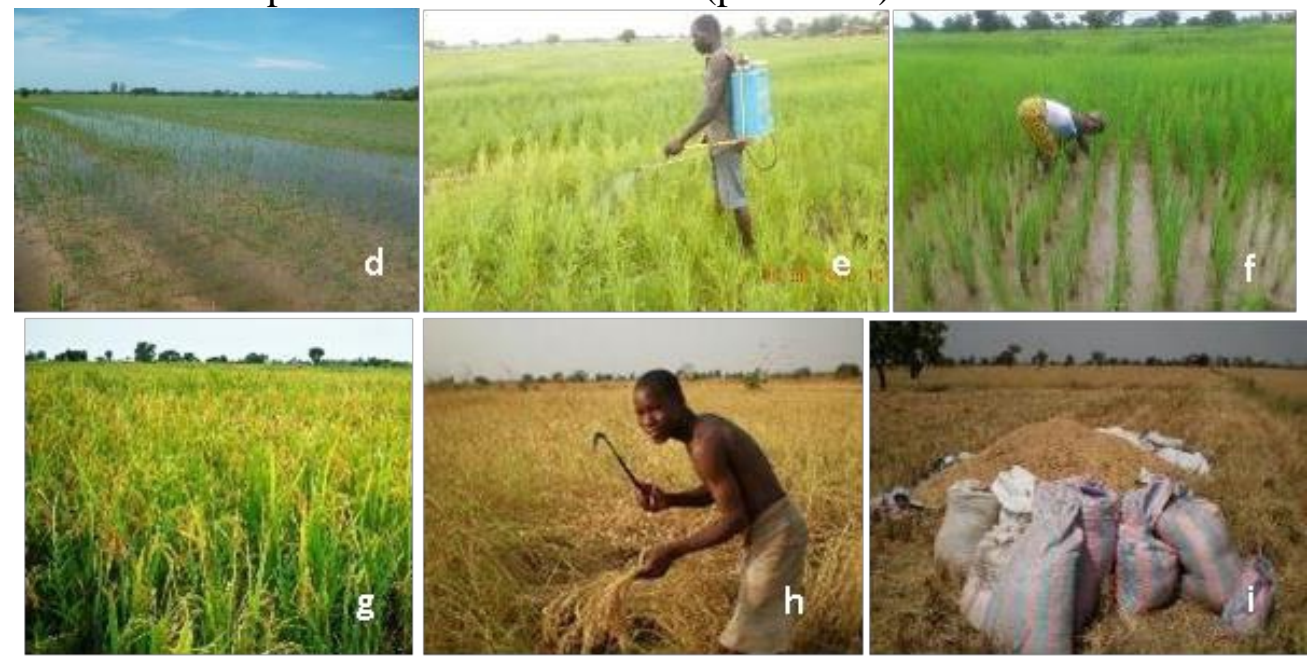

Planche 3. Itinéraire des techniques de la culture du riz au sud du bassin versant de l'Oti Prises de vues: Souberou, travaux de terrain 2014-2015 
La planche 3 résume l'itinéraire technique de la production du riz, de la préparation du sol (photos $3 \mathrm{a}$ et $\mathrm{b}$ ), au semis, (photo $3 \mathrm{c}$ ), à l'épandage de fumures minérales et entretien (photo $3 \mathrm{e}$ ), au sarclage, (photo $3 \mathrm{f}$ ) et au récolte (photos 3g, h et i).

Le maraîchage dans cette aire est pratiqué pour prolonger la campagne agricole. Elle se réalise en deux périodes à savoir la production dite de saison (octobre à décembre) pratiquée sur des sols plats et la production de primeur (janvier à mars). La deuxième période est pratiquée dans les bas-fonds aménagés (maitrise partielle ou totale de l'eau) avec l'appui des projets de développement qui font des renforcements de capacité aux producteurs de tomate (solanum lycopersicum), de piment (Capsicum annuum), de gombo (abelmoschus esculentus) etc., par la formation et bien d'autres actions visant à améliorer la production de ces différentes spéculations.

La rotation des cultures ainsi que l'emploi des cultures de couverture, d'engrais verts, d'engrais organiques et de composts aident à maintenir une bonne structure des sols et un bon équilibre des éléments nutritifs. Des fois, les amendements organiques ne sont pas suffisants pour les cultures et il faut y ajouter des nutriments supplémentaires (Azote, Phosphore, Potassium) afin d'éviter les troubles ou carences chez la plante.

L'association des cultures est pratiquée par 87,63\% des producteurs interviewés dans les bas-fonds non aménagés. Sur une même parcelle trois à quatre cultures ou plus sont combinés pour optimiser toute la durée de la saison. Les cultures les plus associées sont le riz (Oriza sativa), maïs (Zea mays), sorgho (Sorghum bicolor), petit mil (Pennisetum glaucum), l'igname (Dioscorea sp.), patate douce (Solanum tuberosum). Sur d'autres exploitations, ces cultures sont associées aux produits maraîchers tels que le gombo, la tomate, le piment, etc. Par contre, dans les bas-fonds aménagés, les versants sont utilisés pour la culture du maïs (planche 4).
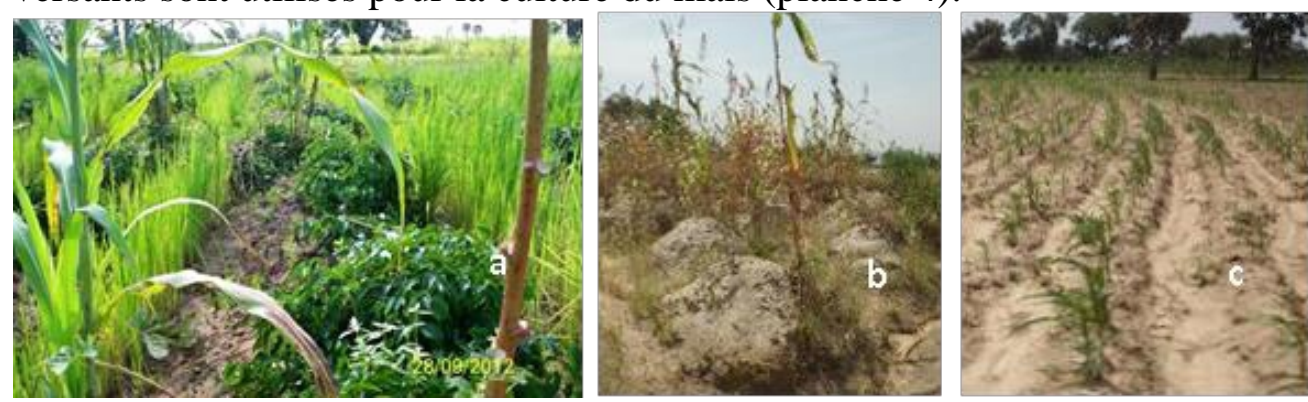

Planche 4. Champs de riz en association avec igname et le sorgho (a) ; Butte d'igname en association avec le riz, le gombo et le sorgho dans les bas-fonds de Alayomdé (b) et Awotobi (Ouaké); maïsiculture sur le versant du bas-fond de Kataban (c) dans le Copargo Prises de vues : Souberou, septembre et novembre 2015

L'observation de la planche 4 révèle que l'association de cultures permet d'occuper le sol avec plusieurs plantes à cycles différents. Ce système 
de diversification de la production mise en place est caractérisé par l'utilisation des matériels traditionnels d'exploitation, le type de main d'œuvre agricole, l'encadrement technique et l'appui assurés par les institutions et les structures de micro-finance (crédits agricoles). Les techniques culturales mises en pratique par les exploitants des bas-fonds lors de la préparation de leurs champs sont le nettoyage et la conservation des résidus de récoltes sur leurs exploitations (100\% des enquêtés), l'essouchage, l'incinération des arbres et brûlages des herbes (lors de la première préparation des champs ou de l'agrandissement de la superficie à exploiter) avant le labour. Ces informations issues de l'enquête de terrain ont montré que la mise en valeur des bas-fonds ces dernières décennies par la production d'une gamme variée de produits agricoles est une réponse suscitée par un certain nombre de facteurs naturels et humains.

\section{Facteurs générateurs de la dynamique de l'exploitation des bas-fonds au sud du bassin versant de l'Oti}

Considérant de plus en plus l'ampleur de la valorisation des bas-fonds mise en exergue plus haut, cette partie examine les causes fondamentales de leur occupation. Il s'agit d'une analyse qui intègre l'explosion démographique, la récurrence des aléas climatiques, la dégradation des sols des plateaux et la richesse pédologique de ses agroécosystèmes parmi les facteurs qui induisent l'exploitation des bas-fonds.

\section{Dynamique spatio-temporelle de la population}

La Figure 2 présente l'évolution de la population de 1979 à 2013 et sa projection aux horizons 2030 et 2050. De 356401 habitants en 1979 (RGPH 1), cet effectif est passé respectivement à 409176 habitants en 1992 (RGPH 2) ; 507609 habitants en 2002 (RGPH 3) et 723760 habitants en 2013 (RGPH 4). Ainsi, cette population a connu un taux d'accroissement annuel de $3,56 \%$ entre 1979 et 1992 . Entre 1992 et 2002, ce taux est passé respectivement de $3,35 \%$ à 3,08 \% entre 2002 et 2013 (INSAE, 2014). La projection faite sur la base de ce taux d'accroissement indique que la population passera à 1267280 habitants en 2030 et à 2294461 habitants en 2050 . 


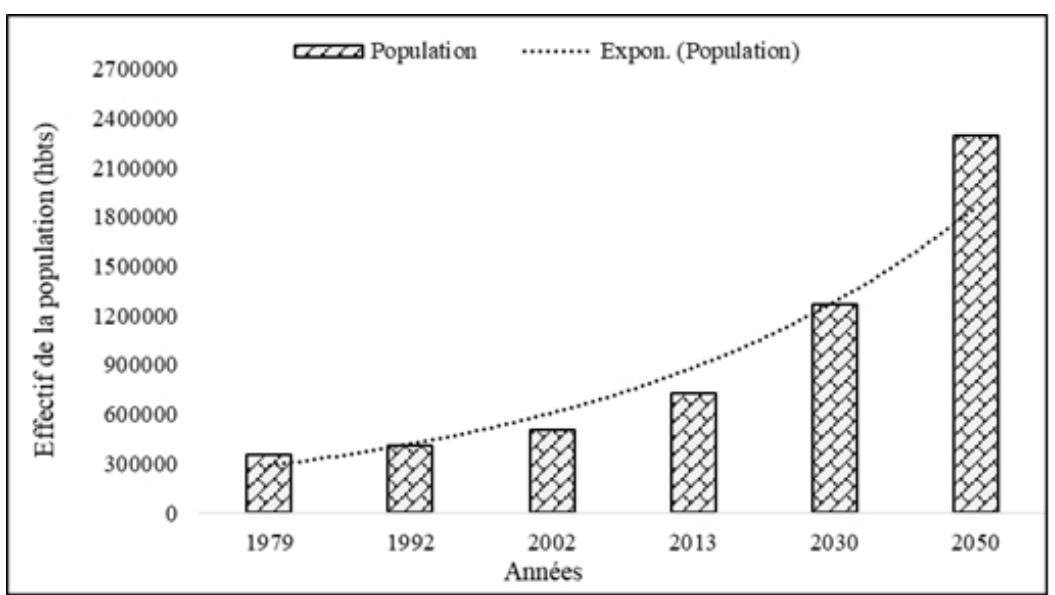

Figure 2. Evolution de la population au sud du bassin versant de l'Oti de 1979 à 2050

Cette population a donc presque doublé entre 1992 et 2013 et va quadruplée en 2050, ce qui constitue un surplus de bouches à nourrir. Par conséquent une augmentation des superficies à cultiver engendrera la déforestation, la reprise agricole des jachères et la mise en culture des basfonds. Ce résultat confirme ceux de Edieti (2012), Issa (2012), Ouorou Barrè (2014) et Wokou (2014) qui ont montré que cette croissance accélérée de la population constitue une force pour la production agricole. Il s'ensuivra une augmentation de la densité de la population à ces mêmes horizons (Figure 3).

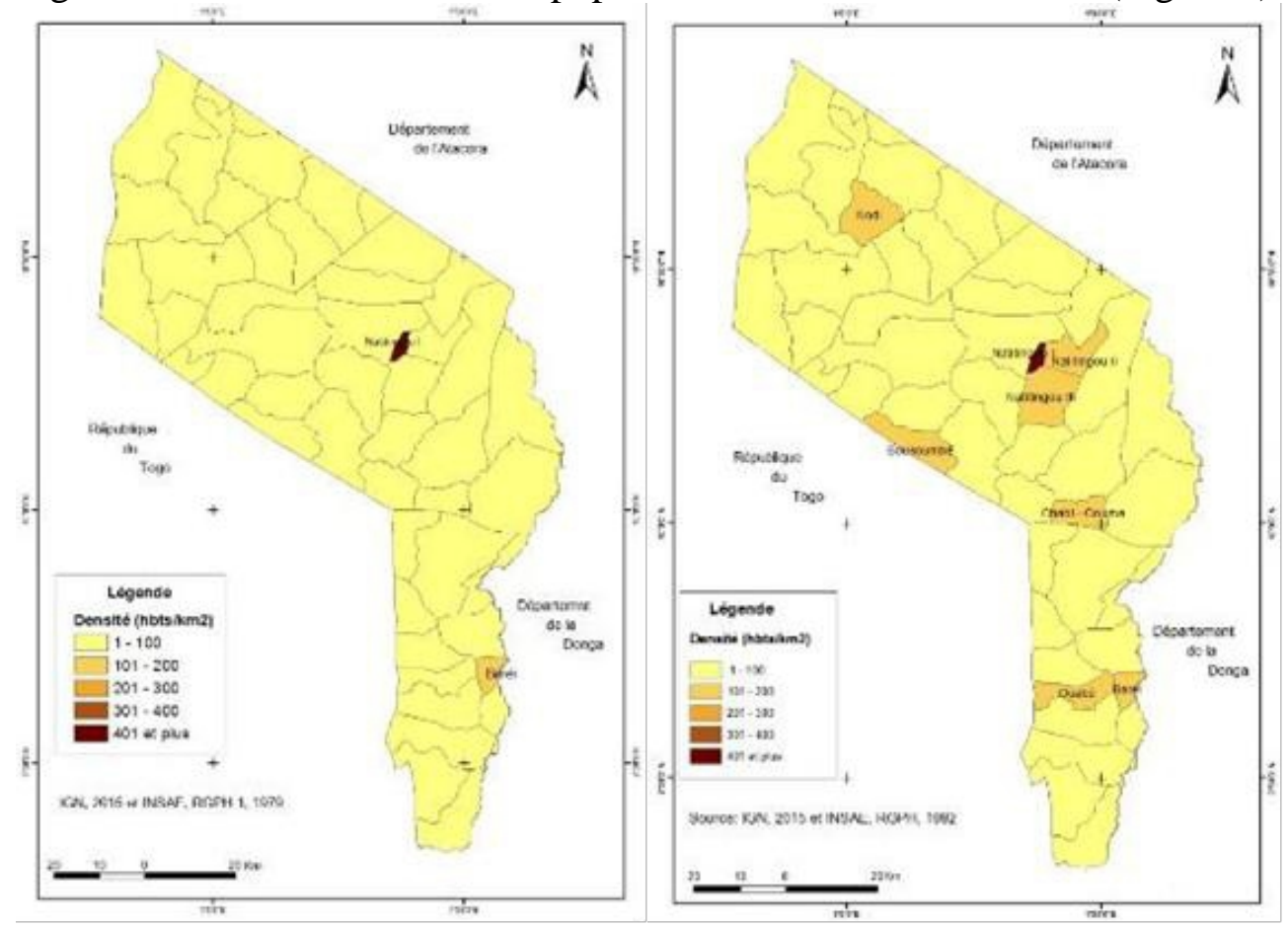



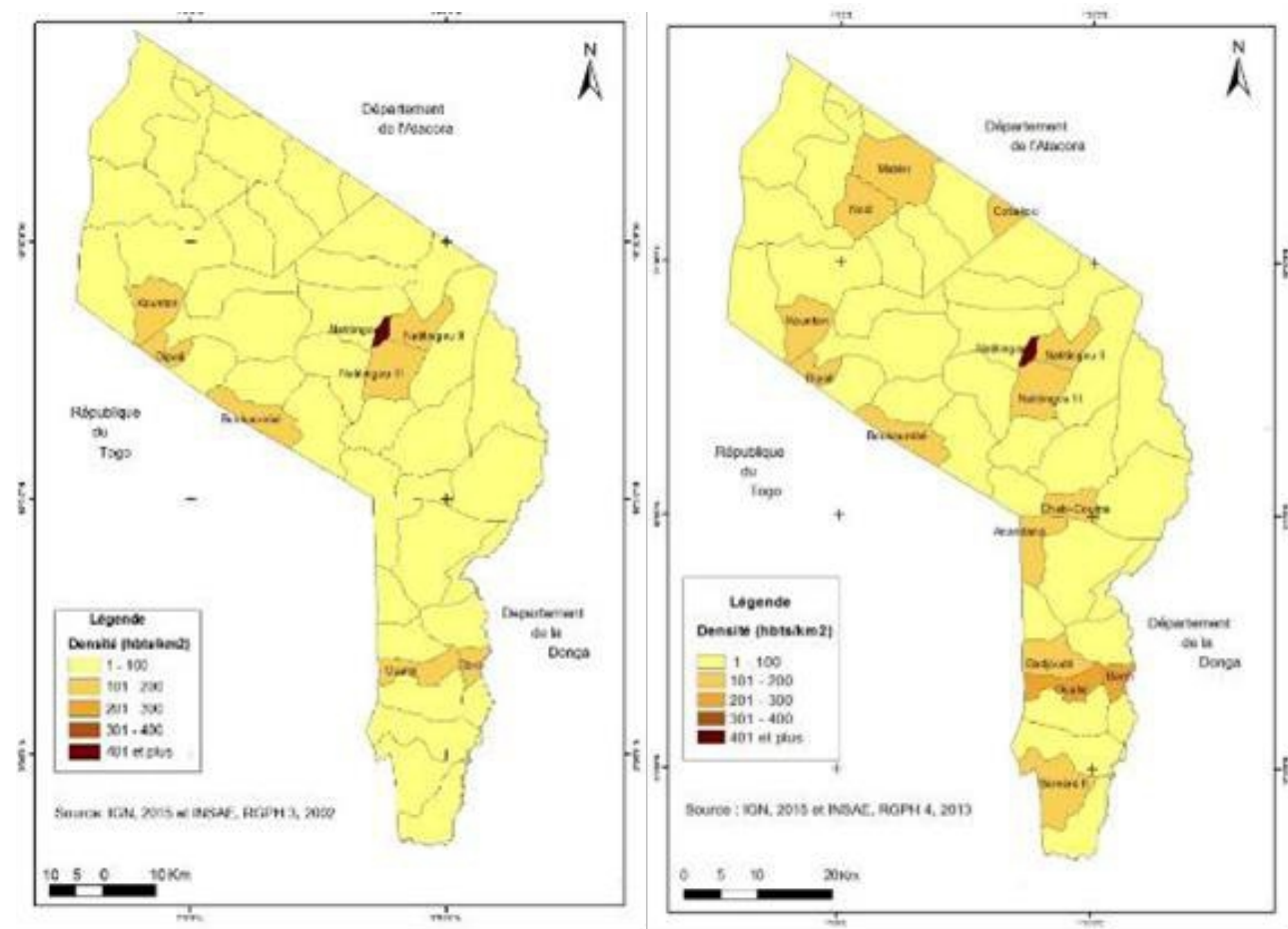

Figure 3. Densité de la population de 1979 à 2013 au sud du bassin versant de l'Oti

L'analyse de la Figure 3 montre que la densité de la population varie entre 1 et 448 habitants au $\mathrm{km}^{2}$ au sud du bassin de l'Oti. De façon spécifique, on constate qu'en 1979, hormis l'arrondissement de Baréi qui avait une densité de 124,39 habitants $/ \mathrm{km}^{2}$, tous les autres Arrondissements avaient une densité de population de l'ordre de 1 à 100 habitants $/ \mathrm{km}^{2}$. En 1992, 2002 et 2013, le nombre d'Arrondissements ayant moins de 100 habitants au $\mathrm{km}^{2}$ a diminué parce que d'autres Arrondissements ont vu leur densité augmenté de 201 à 300 habitants $/ \mathrm{km}^{2}$. L'augmentation de densité de la population entraîne une dynamique dans l'occupation et l'utilisation des sols notamment une pression foncière tout comme la récurrence des aléas climatiques. Ce constat a été observé par Wokou (2014) dans le bassin versant du zou au Bénin.

\section{Evolution des paramètres climatiques}

L'étude de la variation des indices pluviométriques par stations a permis d'analyser l'évolution des précipitations au sud du bassin versant de l’Oti sur la période de 1971-2014 (Figure 4). 


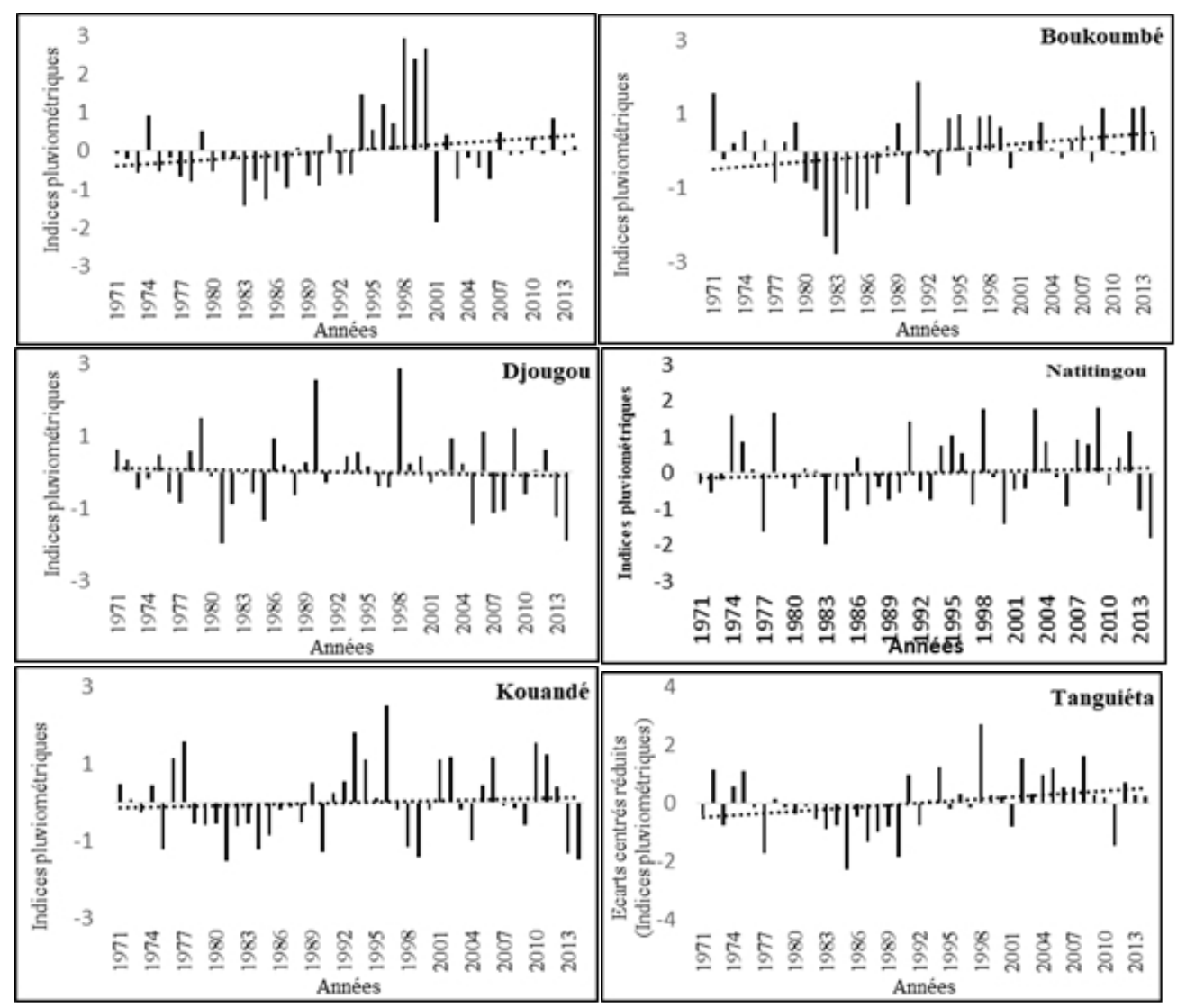

Figure 4. Evolution interannuelle des hauteurs pluviométriques au sud du bassin versant de l'Oti sur la 1971-2014

La période 1971 à 2014 est marquée par une alternance des années pluvieuses et déficitaires de 1971 à 1980, une forte fréquence d'années déficitaires de 1981 à 1991, une forte fréquence d'années pluvieuses de 1992 à 2001 et encore une alternance des années pluvieuses et déficitaires de 2002 à 2014. Sauf au niveau des stations de Boukoumbé et Tanguiéta qui se caractérisent par une forte fréquence d'années pluvieuses pour la dernière séquence (2002-2014). L'alternance d'années pluvieuses et déficitaires a des répercussions sur les activités agricoles (Ouorou Barrè, 2014). Sur ce, le nombre d'années excédentaires, normales et déficitaires est présenté au tableau I par station. 
Tableau I. Typologie des années entre 1971 et 2014

\begin{tabular}{|c|c|c|c|c|c|}
\hline \multirow[t]{2}{*}{ Stations } & \multicolumn{5}{|c|}{ Types d'années } \\
\hline & Excédentaires & $\begin{array}{c}\text { Très } \\
\text { excédentaires } \\
\end{array}$ & Normales & déficitaires & Très déficitaires \\
\hline \multirow[t]{2}{*}{ Boukoumbé } & $\begin{array}{l}1974 ; 1979 ; 1989 ; \\
1994 ; 1995 ; 1997 ; \\
1998 ; 1999 ; 2003 ; \\
2007\end{array}$ & $\begin{array}{l}1971 ; 1991 ; \\
2009 ; 2012 \\
2013\end{array}$ & $\begin{array}{l}\text { 1972; 1973; 1975; 1976; } \\
\text { 1978; 1988; 1992; 1996; } \\
\text { 2000; 2001; 2002; 2004; } \\
\text { 2005; 2006; 2008; 2010; } \\
\text { 2011; 2014 }\end{array}$ & $\begin{array}{l}1977 ; 1980 \\
1981 ; 1987 \\
1993\end{array}$ & $\begin{array}{l}1982 ; 1983 \\
1984 ; 1985 \\
1986 ; 1990\end{array}$ \\
\hline & $22,73 \%$ & $11,36 \%$ & $40,91 \%$ & $11,36 \%$ & $13,64 \%$ \\
\hline \multirow[t]{2}{*}{ Djougou } & $\begin{array}{l}1971 ; 1978 ; 1986 \\
1994 ; 2003 ; 2012\end{array}$ & $\begin{array}{l}1979 ; 1990 \\
1998 ; 2006 \\
2009\end{array}$ & $\begin{array}{l}\text { 1972; 1973; 1974; 1975; } \\
\text { 1980; 1983; 1987; 1989; } \\
\text { 1991; 1992; 1993; 1995; } \\
\text { 1996; 1997; 1999; 2000; } \\
\text { 2001; 2002; 2004; 2011 }\end{array}$ & $\begin{array}{l}1976 ; 1977 \\
1982 ; 1984 \\
1988 ; 2010\end{array}$ & $\begin{array}{l}1981 ; 1985 \\
2005 ; 2007 ; \\
2008 ; 2013 ; \\
\quad 2014\end{array}$ \\
\hline & $13,64 \%$ & $11,36 \%$ & $45,45 \%$ & $13,64 \%$ & $15,91 \%$ \\
\hline \multirow[t]{2}{*}{ Kouandé } & 1989; 1992; & $\begin{array}{l}1976 ; 1977 ; \\
\text { 1993; 1994; } \\
\text { 1996; 2001; } \\
\text { 2002; 2006; } \\
\text { 2010;2011 }\end{array}$ & $\begin{array}{l}1971 ; 1972 ; 1973 ; 1974 ; \\
\text { 1986; 1987; 1991; 1995; } \\
\text { 1997; 2000;2003; 2005; } \\
\text { 2007; 2008; } 2012\end{array}$ & $\begin{array}{c}1978 ; \\
1979 ; 1980 \\
1982 ; 1983 ; \\
1985 ; 1988 \\
2004 ; 2009\end{array}$ & $\begin{array}{l}1975 ; 1981 ; \\
1984 ; 1990 ; \\
1998 ; 1999 ; \\
2013 ; 2014\end{array}$ \\
\hline & $4,54 \%$ & $22,73 \%$ & $34,09 \%$ & $20,46 \%$ & $18,18 \%$ \\
\hline \multirow[t]{2}{*}{ Matéri } & $\begin{array}{c}\text { 1974; 1979; 1995; } \\
1997 ; 2012\end{array}$ & $\begin{array}{l}1994 ; 1996 ; \\
1998 ; 1999 \\
2000\end{array}$ & $\begin{array}{c}1971 ; 1972 ; 1976 ; 1981 ; \\
1982 ; 1988 ; 1991 ; 2002 ; \\
2004 ; 2005 ; 2007 ; 2008 ; \\
2009 ; 2010 ; 2011 ; 2013 ; \\
2014\end{array}$ & $\begin{array}{l}\text { 1973; 1975; } \\
\text { 1977; 1978; } \\
\text { 1980; 1984; } \\
\text { 1986; 1987; } \\
\text { 1989; 1990; } \\
\text { 1992; 1993; } \\
\text { 2003; 2006; }\end{array}$ & $\begin{array}{l}\text { 1983; 1985; } \\
2001\end{array}$ \\
\hline & $11,36 \%$ & $11,36 \%$ & $38,63 \%$ & $31,83 \%$ & $6,82 \%$ \\
\hline \multirow[t]{2}{*}{ Natitingou } & $\begin{array}{c}\text { 1975; 1994; 1995; } \\
\text { 1996; 2004; 2007; } \\
\text { 2008; }\end{array}$ & $\begin{array}{l}1974 ; 1978 ; \\
1991 ; 1998 ; \\
2003 ; 2009 ; \\
2012\end{array}$ & $\begin{array}{l}1971 ; 1972 ; 1973 ; 1976 ; \\
1979 ; 1980 ; 1981 ; 1982 ; \\
1984 ; 1986 ; 1987 ; 1988 ; \\
1989 ; 1997 ; 1999 ; 2002 ; \\
2005 ; 2010 ; 2011\end{array}$ & $\begin{array}{l}1985 ; 1990 ; \\
1992 ; 1993 ; \\
2001 ; 2006 ; \\
\quad 2013\end{array}$ & $\begin{array}{l}1977 ; 1983 ; \\
2000 ; 2014\end{array}$ \\
\hline & $15,91 \%$ & $15,91 \%$ & $43,18 \%$ & $15,91 \%$ & $9,09 \%$ \\
\hline \multirow[t]{2}{*}{ Tanguiéta } & $\begin{array}{l}\text { 1974; 1991; 2004; } \\
\text { 2006; 2007; } 2012\end{array}$ & $\begin{array}{l}1972 ; 1975 \\
\text { 1994; 1998; } \\
\text { 2002; 2005; } \\
\quad 2008\end{array}$ & $\begin{array}{c}1971 ; 1978 ; 1979 ; 1980 ; \\
\text { 1981; 1986; 1993; 1995; } \\
\text { 1996; 1997; 1999; 2000; } \\
\text { 2003; 2009; 2010; 2013; } \\
2014 \\
\end{array}$ & $\begin{array}{c}1973 ; 1976 ; \\
1982 ; 1983 \\
1984 ; 1988 \\
1989 ; 1992 \\
2001 \\
\end{array}$ & $\begin{array}{l}1977 ; 1985 ; \\
1987 ; 1990 \\
2011\end{array}$ \\
\hline & $13,64 \%$ & $15,91 \%$ & $38,63 \%$ & $20,46 \%$ & $11,36 \%$ \\
\hline $\begin{array}{l}\text { Moyenne des } \\
\text { pourcentages }\end{array}$ & $13,64 \%$ & $14,77 \%$ & $40,15 \%$ & $18,94 \%$ & $12,5 \%$ \\
\hline
\end{tabular}

Source : Traitement des données climatiques, juin, 2015

Les années humides, normales et sèches enregistrées sont hétérogènes d'une station à l'autre. Considérant toutes les stations ensemble, l'année «1983» est une année sèche au sud du bassin versant de l'Oti et l'année «1994» est une année pluvieuse, commune à toutes les stations. Il faut signaler qu'au cours de la période d'étude, il est observé des successions d'années sèches (stations de Boukoumbé, Kouandé, Matéri et Tanguiéta) et des années 
humides (stations de Matéri, Natitingou et Tanguiéta). Globalemenent le milieu d'étude a enregistré 14,77 \% d'années très pluvieuses, 13, $64 \%$ d'années pluvieuses, 40,15\% d'années normales, 18,94\% d'années sèches et $12,5 \%$ d'années très sèches. De cette analyse climatique, 59,85\% des années de 1971 à 2014 ont constitué des contraintes majeures pour l'agriculture notamment des répercussions sur les activités agricoles au sud du bassin versant de l'Oti. Ce taux se rapproche de celui de Ouorou Barrè (2014) dans le Département de l'Atacora, soit de $66 \%$ des années de 1971 à 2010 lors de l'analyses de la variation interannuelle des hauteurs pluviométriques. Les problèmes agricoles (engorgement des sols, pourriture des racines, asphyxie des plantes par manque d'oxygène) se sont posées pour les cultures de cycle court et de faible enracinement à cause de l'excès des précipitations continues, notamment pour le maïs et le riz. Par conséquent les rendements agricoles ont été affectés malgré les hauteurs de pluies enregistrés au cours des périodes humides.

Les producteurs ont eu recours à une irrigation d'appoint des cultures ou opter pour la riziculture des bas-fonds (avec maîtrise partielle ou totale de l'eau, repiquage des plants) puisqu'il va falloir couvrir les besoins en eau des cultures pour éviter qu'il y ait un effet de stress hydrique chez les plantes. Donc la mise en valeur des bas-fonds par des aménagements hydroagricoles adéquats pour la gestion des excédents, constitue une option afin de lever cette situation défavorable aux cultures.

\section{Dégradation des sols des plateaux}

La dégradation des sols sur les plateaux au sud du bassin versant de l'Oti est aussi l'une des facteurs influençant la dynamique d'occupation des bas-fonds. Ces quatre dernières décennies, il a été noté une diminution des terres agricoles cultivable sur les interfluves et autres parties du bassin, sous la double implication de l'augmentation des actifs agricoles et de l'augmentation des superficies (déforestation, reprise agricole de jachère) pour accroitre les productions agricoles. Selon les investigations faites sur le terrain, la superficie moyenne annuelle défrichée en hautes altitudes varie de 0 à 4,8 ha et la majorité des exploitants $(67,86 \%)$ défriche entre 0 et 1 ha dans des bas-fonds comme alternative à l'épuisement tendanciel de la fertilité des sols des hauts plateaux.

Le système actuel de production est peu performant et nécessite cinq à dix années de jachère. Les principales cultures sont le sorgho, le mil, le maïs, le manioc, l'igname, le fonio, l'arachide, le riz et le niébé. Ces systèmes qui sont souvent des associations, surchargent les parcelles et ne permettent pas l'utilisation rationnelle des intrants. Il s'en est suivi un appauvrissement rapide des sols et un exode massif des populations. 
Avec l'impossibilité de trouver des terres cultivables dans les parties en hautes altitudes et en raison des contraintes climatiques contemporaines, les producteurs ont orientés le front agricole (cultures céréalières, tubercules et cultures maraîchères pratiquées en monoculture et en association) vers les bas-fonds en raison de leur relative fertilité et surtout de la permanence de l'eau. Cette pratique courante au sud du bassin versant de l'Oti s'accentue en réponse aux impacts négatifs de la variabilité climatique, de la baisse de la fertilité des sols et de l'explosion démographique.

Le niveau de dégradation alarmant des sols sur les plateaux et le souci de diversification agricole et de revenus ont donc amené les producteurs à conquérir d'autres espaces cultivables. Ceci confirme la tendance à la hausse de la superficie mise en valeur dans les bas-fonds et de la production de la culture riz de 1995 à 2014 (Figure 8).

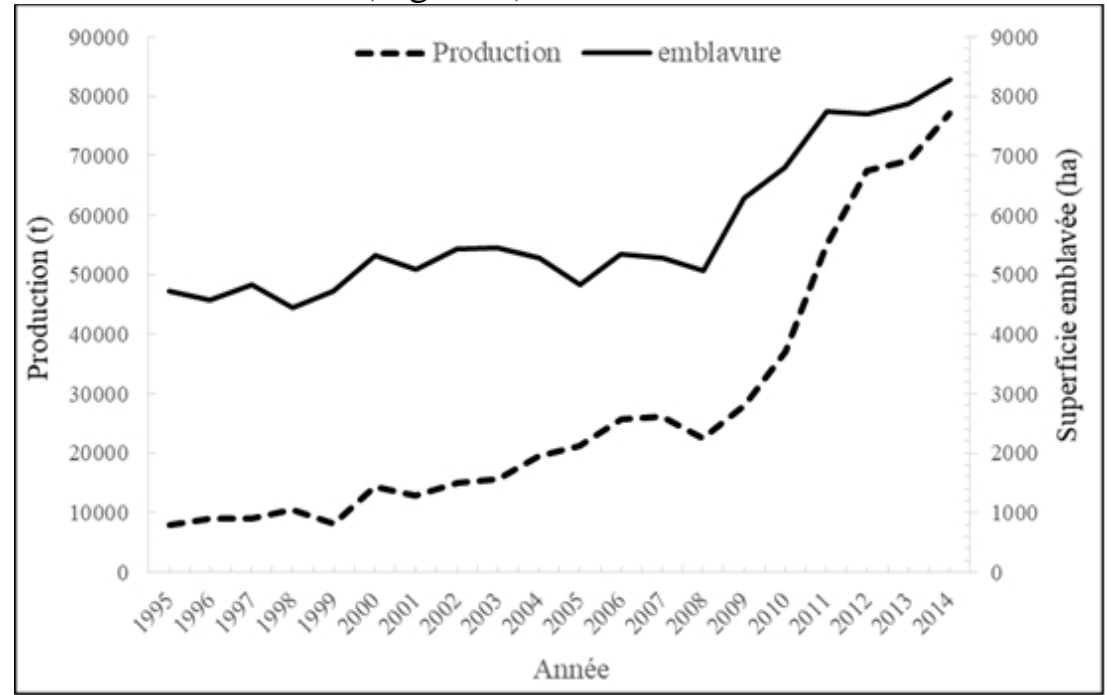

Figure 8. Evolution de l'emblavure et de la production du riz de 1995 à 2014 au sud du bassin versant de l'Oti

La Figure 8 montre qu'au cours de la période d'analyse, la superficie emblavée pour la culture du riz (Oriza sativa) a continuellement augmenté et est marquée par une nette évolution exponentielle à partir de l'année 2008. Cette augmentation des emblavures confirme l'évolution ascendante de la production avec un taux moyen annuel de production de $13 \%$.

Ainsi, les contraints climatiques, la baisse de fertilité des sols agricoles en haute altitude et les mauvaises pratiques agricoles induisent la dégradation des terres et justifient l'augmentation des superficies cultivées.

En effet, dans le souci d'augmenter la production agricole en conformité avec leurs besoins, de nouvelles terres sont mises en valeur y compris les bas-fonds, ce qui engendre des changements environnementaux. Selon plus 96,83\% des interviewés qui investissent dans les bas-fonds, leurs 
sols sont considérés comme fertile et humides pendant plusieurs mois de l'année. Il s'agit des sols hydromorphes et sols ferrugineux tropicaux (hydromorphes ; lessivés sans concrétions ou à concrétions ; indurés). Ce sont des sols peu profonds $(0-120 \mathrm{~cm})$ possédant un bon potentiel de fertilité et d'aptitude à l'agriculture (photo 1). Le drainage sur ces sols est faible et l'inondation est fréquente sur les sols hydromophes et peu fréquente sur les sols ferrugineux en période culturale. Ils disposent d'une quantité très élevée de matière organique mais la teneur en azote est moyenne.Difficiles à travailler, ces sols sont plastiques quand ils sont humides et durs quand ils sont secs. Leurs exploitations nécessitent des techniques culturales appropriées.

\section{Perceptions paysannes des facteurs conduisant à la mise en valeur des bas-fonds}

$\mathrm{Au}$ sud du bassin versant de l'Oti, nombreux sont les producteurs agricoles qui investissent dans la valorisation des bas-fonds. Les opinions exprimées par la population révèlent que la mise en valeur des bas-fonds est liée à la baisse des hauteurs de pluie ou à leur variation, à la longue période de la saison sèche et à la succession de deux voire plus de trois années sèches (Figure 9).

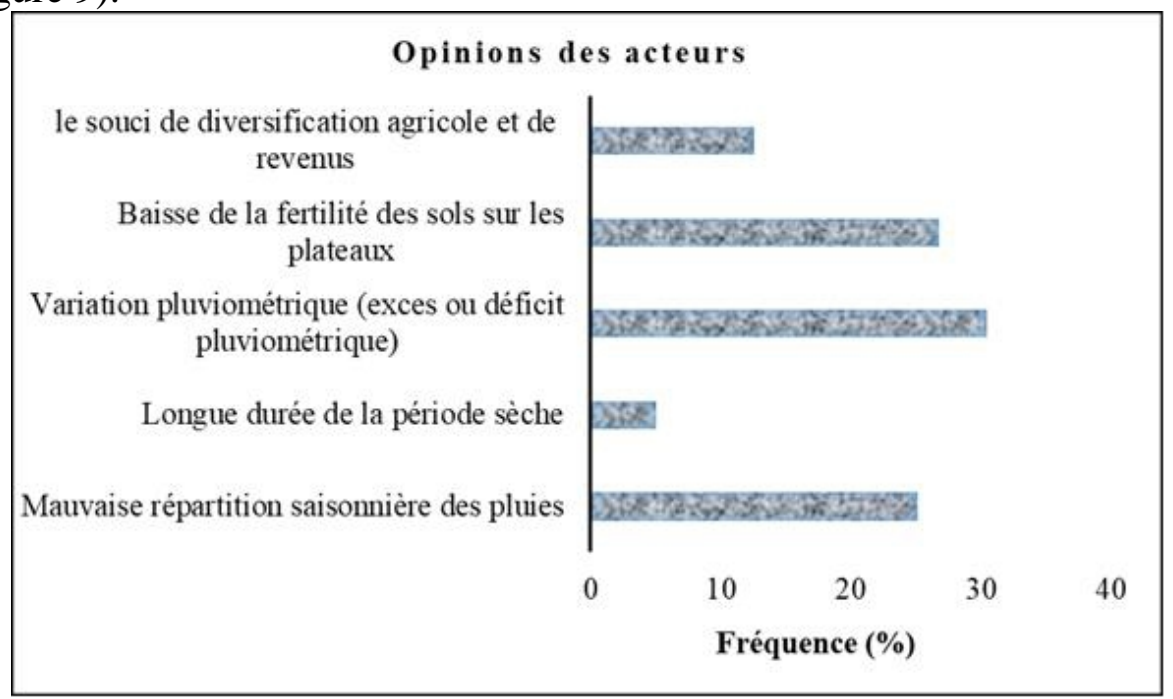

Figure 9. Opinions endogènes des acteurs sur les raisons de la mise en valeur des bas-fonds Source : Enquête de terrain, 2015

De l'analyse de la Figure 9, plus de $60 \%$ et $12 \%$ des enquêtés exploitent les bas-fonds respectivement lorsqu'il y a une perturbation du cycle pluviométrique affectant ainsi le système de culture et dans le souci de diversification et de revenus agricoles.

Aussi, les personnes interviewées $(95 \%)$ ont révélés que ce facteur lié aux variabilités climatiques prend une part active au processus de la 
dynamique d'occupation et d'utilisation des bas-fonds. Ces perceptions recueillies sur le bassin versant de l'Oti est en phase avec celles rapportées dans la littérature (Ouédraogo et al., 2010 ; Souberou, 2013 ; Idani, 2014) puis qu'elles soulignent toutes un déficit pluviométrique. A ceci, s'ajoute l'augmentation des actifs agricoles de ces quatre dernières décennies qui a entrainé une augmentation des emblavures engendrant la diminution des terres cultivables sur les interfluves. Plus grave, les différents types de sols sont en proie à la dégradation du fait des pratiques culturales occasionnant la baisse de la fertilité du sol selon Ouorou Barrè (2014).

La présence prolongée de l'humidité du sol et la fertilité du sol des basfonds sont d'autres raisons qui orientent les interviewés (98\%) vers l'exploitation des bas-fonds pour une bonne production agricole. La mise en valeur des bas-fonds s'impose et constitue une stratégie d'adaptation dans ce contexte de changements climatiques, en dehors d'une réponse suscitée par la dégradation des sols des plateaux, la réduction progressive de la durée de jachère due à la pression démographique. Il importe donc de comprendre que la baisse de la pluviométrie provoque une concentration des diverses cultures dans les bas-fonds où le taux d'humidité du sol est plus élevé que sur les terres hautes (Albergel et al., 1993). Cette analyse est confirmée par Dembelé (2010) dans son étude qui pense que l'engouement pour la mise en valeur des basfonds est accentué par la croissance des actifs agricoles, la persistance de la péjoration climatique et la satisfaction du marché (facteur économique). Donc la mise en valeur des bas-fonds est une riposte à la pression foncière sur les terres hautes, un moyen de sécurisation ou accroissement de la production agricole et un moyen pour la gestion efficiente des ressources naturelles (Souberou et al., 2017).

\section{Conclusion}

La dynamique active de l'occupation et de l'utilisation des bas-fonds pour la production d'une gamme variée de cultures (riz, mais, sorgho, mil, igname, etc.) est induite par la dynamique croissance des actifs agricoles de 1979 à 2013 entrainant une extension des espaces agricoles, à la récurrence des aléas climatiques de 1971 à 2014 (variation des paramètres climatique), à la dégradation des sols en hautes altitudes (dû à un certain nombre de pratiques culturales utilisé, entre autre la culture itinérante sur brulis, le réduction du temps de la jachère, la mauvaise gestion des sols et des eaux, etc.). Il ressort également de cette étude que la fertilité des sols des bas-fonds et la présence prolongée de l'humidité sont d'autres raisons qui orientent les interviewés (98 $\%$ ) vers l'exploitation des bas-fonds pour une bonne production agricole

La mise en valeur des bas-fonds est une activité économiquement rentable et nécessite l'implantation des ouvrages hydroagricoles afin de lever les limites et les contraintes d'exploitation. Ils constituent dans ce contexte de 
changements climatiques et de mutation des modes d'utilisation des sols agricoles, des surfaces de très grand intérêt et importance.

\section{References:}

1. Abdou, B.A. (2016). Perceptions et adaptation des producteurs agricoles à la variabilité climatique et ses impacts dans la vallée de la Gorama à Gouna (Niger), In Actes du Colloque en hommage aux Professeurs Michel BOKO et Fulgence AFOUDA sur le thème 'Risques et catastrophes climatiques : Vulnérabilité et adaptation en Afrique de l'Ouest, Volume 2 : Agro-climatologie, 18 pp.

2. Agbossou, K.E., Hounsou, B.M., \& Ahamidé, B. (2004). Essai de typologie des bas-fonds de la commune de Cové du Sud-Bénin.Thème 4 : Bas-fonds : Communication 1, 17p

3. Albergel, J., Lamachere, J.M., Lidon, B., Mokadem, A.I., \& Vandriel, W. (1993). Mise en valeur agricole des bas-fonds du Sahel, Typologie, fonctionnement hydrologique, potentialités agricoles. Rapport final d'un projet (CORAF-R3S), Cirad, Montpellier, France, 335p.

4. Dembélé, Y. (2010). Intérêt du développement des bas-fonds en Afrique de l'Ouest: Implication des bénéficiaires et inventaire du potentiel, Atelier de clôture du projet RAP Phase 1 (2009-2010), 7 au 9 décembre 2010, INERA/Burkina Faso

5. Durand, C. (2002). Méthodes de sondage : L'échantillonnage et la gestion du terrain. Note de cours, Département de sociologie, Université de Montréal, $77 \mathrm{p}$.

6. Idani, M. (2014). Indicateurs environnementaux de prévision et de gestion des catastrophes climatique par les Berba de Matéri. Master en Gestion des risques et catastrophes. MIRD/FLASH/UAC, 86 p.

7. Idieti, E.M. (2012). Les hydro-écorégions du Bassin de la Pendjari au Bénin : Analyse des déterminants socio-économiques et environnementaux de la dynamique des écosystèmes naturels. Thèse de doctorat de géographie. Université d'Abomey-Calavi, 229 p.

8. INSAE [Institut National de la Statistique et de l'Analyse Economique] (2014). Résultats provisoires du recensement général de la population et de 1'habitation. (RGPH4). Cotonou, $47 \mathrm{p}$.

9. INSAE [Institut National de la Statistique et de l'Analyse Economique] (2005). Cahiers des villages et quartiers de ville du Département de 1'Atacora INSAE, Cotonou $25 \mathrm{p}$.

10. Issa, M.S. (2012). Changements climatiques dans le moyen Bénin : impacts, analyse prospective des agro-systèmes et mesures d'adaptation/alternatives. Thèse de doctorat unique, EDP/FLASH, $\mathrm{UAC}, 250 \mathrm{p}$. 
11. Lavigne Delville, PH., Boucher, L., \& Vidal, L. (1996). Les bas-fonds en Afrique tropicale humide : stratégies paysannes, contraintes agronomiques et aménagements" in Pichot et al eds. Fertilité du milieu et stratégies paysannes sous les tropiques humides, actes du séminaire international, CIRAD, pp. 148-161.

12. Mbodj, S. (2008). Une meilleure valorisation des ressources des basfonds du Sine Saloum par la GIRE. Expérience du Programme de Lutte contre la Pauvreté en milieu rural dans le Bassin Arachidier 2004 2007, Programme sénégalo-allemand d'appui à la décentralisation et au développement local PRODDEL, 88 p.

13. Ouedraogo, M., Dembelé, Y., \& Somé, L. (2010). Perceptions et stratégies d'adaptation aux chamgements des précipitations : cas des paysans du Burkina-Faso. Sécheresse 21 (2) : 87-96.

14. Ouorou Barrè, F.I. (2014). Contraintes climatiques, pédologiques et production agricole dans l'Atacora (Nord-Ouest du Benin). Thèse de doctorat unique, EDP/FLASH, UAC, $241 \mathrm{p}$.

15. Schwartz, D. (1995). Méthodes statistiques à l'usage des médecins et

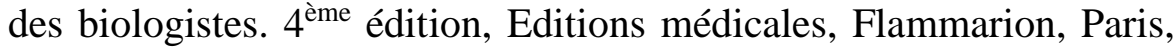
$314 \mathrm{p}$.

16. Souberou, K. T., Toko Mouhamadou, I., Ogouwale, E., \& Agbossou, K. E. (2017). Dynamics of the Inland Valley Occupation in the Oti Watershed in Benin: Diagnosis and Prospective Analysis, in International Journal of sciences, Volume 6 - December 2017 (12), Published at: http://www.ijsciences.com/pub/issue/2017-12/ DOI: 10.18483/ijSci.1484; Online ISSN: 2305-3925; Print ISSN: 24104477.

17. Souberou, K.T. (2013). Contribution de la Télédétection et du SIG à la caractérisation des bas-fonds de la Commune de Matéri (Nord-Ouest, Bénin). Mémoire de Master en Science de la Géo-Information, Centre Régional de Formation aux Techniques des Levés Aérospatiaux (RECTAS). Université d'Obafèmi Awolowo au Nigéria, , 135 p.

18. Wokou, C. G. (2014). Croissance démographique, évolution climatique et mutations agricoles et environnementales dans le bassin du Zou. Thèse de doctorat unique, EDP/FLASH, UAC, 244p. 